\title{
O-GIcNAcylation contributes to intermittent hypoxia-associated vascular dysfunction via modulation of MAPKs but not CaMKII pathways
}

\author{
XUELING GUO ${ }^{1}$, YAN DENG ${ }^{2}$, LINGHUI ZHAN ${ }^{1}$, JIN SHANG $^{2}$ and HUIGUO LIU ${ }^{2}$ \\ ${ }^{1}$ Department of Critical Care Medicine, Zhongshan Hospital Affiliated to Xiamen University, Xiamen, Fujian 361004; \\ ${ }^{2}$ Department of Respiratory and Critical Care Medicine, Key Laboratory of Respiratory Disease of The Ministry of Health, \\ Tongji Hospital, Huazhong University of Science and Technology, Wuhan, Hubei 430030, P.R. China
}

Received February 1, 2021; Accepted May 28, 2021

DOI: $10.3892 / \mathrm{mmr} .2021 .12384$

\begin{abstract}
Intermittent hypoxia (IH) leads to vascular dysfunction, and O-linked- $\beta$-N-acetylglucosamine (O-GlcNAc)ylation may regulate vascular reactivity through the modulation of intracellular signaling. The present study hypothesized that O-GlcNAc modifications contributed to the vascular effects of acute IH (AIH) and chronic IH (CIH) through the MAPK and $\mathrm{Ca}^{2+} /$ calmodulin-dependent kinase II (CaMKII) pathways. Rat aortic and mesenteric segments were incubated with DMSO, O-GlcNAcase (OGA) or O-GlcNAc transferase (OGT) inhibitor under either normoxic or AIH conditions for $3 \mathrm{~h}$, and arterial function was then assessed. Meanwhile, arteries isolated from control and $\mathrm{CIH}$ rats were exposed to $3 \mathrm{~h}$ of incubation under normoxic conditions using DMSO, OGA or OGT as an inhibitor, before assessing arterial reactivity. $\mathrm{CIH}$ was found to increase the expression of vascular O-GlcNAc protein and OGT, phosphorylate p38 MAPK and ERK1/2, and decrease OGA levels, but it had no effects on phosphorylated CaMKII levels. OGA inhibition increased global O-GlcNAcylation and the phosphorylation of p38 MAPK, ERK1/2 and CaMKII, whereas OGT blockade had the opposite effects. OGA inhibition preserved acetylcholine-induced relaxation in AIH arteries, whereas OGT blockade attenuated the relaxation responses of arteries under normoxic conditions or undergoing AIH treatments. However, the impairment of acetylcholine dilation in $\mathrm{CIH}$ mesenteric arteries was improved. CIH artery contraction was increased following angiotensin II (Ang II) exposure. Blockade of p38 MAPK and
\end{abstract}

Correspondence to: Professor Huiguo Liu, Department of Respiratory and Critical Care Medicine, Key Laboratory of Respiratory Disease of The Ministry of Health, Tongji Hospital, Huazhong University of Science and Technology, 1095 Jiefang Avenue, Wuhan, Hubei 430030, P.R. China

E-mail: hgliu@tjh.tjmu.edu.cn

Key words: O-GlcNAcylation, intermittent hypoxia, vascular reactivity, p38 MAPK, ERK1/2
ERK1/2, but not CaMKII, attenuated Ang II-induced contractile responses in $\mathrm{CIH}$ arteries isolated from the non-OGT inhibitor-treated groups. OGT inhibition significantly blocked contractile responses to Ang II and abolished the inhibitory effects of MAPK inhibitors. These findings indicated that $\mathrm{O}-$ GlcNAcylation regulates $\mathrm{IH}$-induced vascular dysfunction, at least partly by modulating MAPK, but not CaMKII, signaling pathways.

\section{Introduction}

Obstructive sleep apnea (OSA) is a common disorder characterized by episodic arterial oxygen desaturation due to recurrent closure of the upper airway during sleep, namely intermittent hypoxia ( $\mathrm{IH})$, which can trigger excessive oxidative stress, systemic inflammation, sympathetic activation and increased renin generation, leading to abnormal vascular reactivity $(1,2)$. IH-related vascular dysfunction involves impaired acetylcholine $(\mathrm{ACh})$-induced endothelium-dependent relaxation $(3,4)$ and increased contractile responses to vasoconstrictors, such as angiotensin II (Ang II) (5,6) and endothelin-1 (ET-1) (7-9), which underlie the initiation and progression of hypertension. In our previous study, rats exposed to chronic IH (CIH) were found to exhibit enhanced Ang II-induced vasoconstriction, which was possibly attributed to the overactivation of ERK1/2 signaling through the upregulation of myosin phosphatase targeting subunit (MYPT1) and myosin light chain (MLC) phosphorylation (5). Other studies have shown that IH increases ET-1-mediated constriction by increasing Rho-kinase activation $(7,8)$ or through a protein kinase $\mathrm{C}(\mathrm{PKC})$-mitochondrial oxidative pathway (9), leading to the increase in $\mathrm{Ca}^{2+}$ sensitization and sensitivity of vascular smooth contractile machinery.

O-linked- $\beta$-N-acetylglucosamine (O-GlcNAc) modifications on serine/threonine (Ser/Thr) residues of proteins, known as O-GlcNAcylation, are a critical regulatory post-translational modification (PTM), analogous to phosphorylation, that can induce functional changes in numerous target proteins, including protein kinases, phosphatases, cellular signaling proteins and transcriptional factors $(10,11)$. O-GlcNAcylation is controlled by two conserved enzymes, namely O-GlcNAc transferase (OGT) and O-GlcNAcase 
(OGA). OGT catalyzes the addition of O-GlcNAc to the Ser/Thr residues of target proteins, whereas OGA is responsible for the hydrolytic cleavage of O-GlcNAc from post-translationally modified proteins (10). O-GlcNAc modifications actively participate in the regulation of cardiovascular pathological processes, such as hypertension, cardiac hypertrophy, ischemia-reperfusion (I/R) injury and vascular inflammatory injury $(10,12,13)$. In our previous study, it was found that the O-GlcNAc protein and cellular signaling involving ERK1/2 and p38 MAPK may act as potential regulators in $\mathrm{IH}$-related cardiac remodeling and left ventricular function (14). Lima et al (15) reported that global O-GlcNAc proteins were elevated in the arteries of deoxycorticosterone acetate and salt (DOCA-salt)-induced hypertensive rats. Increasing O-GlcNAc levels resulted in impaired endothelium-dependent relaxation and increased reactivity to constrictor stimuli, which are hallmarks of hypertension. A reduction in phosphorylated Akt and endothelial NO synthase (eNOS), possibly due to direct competition with O-GlcNAc, decreased NO production and therefore contributed to vascular dysfunction (15-17). These findings support a contributing role of O-GlcNAcylation in regulating vascular reactivity, which underlies the pathogenesis of hypertension.

Since both O-GlcNAcylation and phosphorylation modify the same or neighboring Ser/Thr residues of substrate proteins, an extensive interplay, termed PTM crosstalk, exists between them, including competitive site occupancy, alternative occupancy at adjacent sites and simultaneous occupancy at the same protein (18). OGA and OGT often occur in the same protein complexes as kinases and phosphatases, suggesting that a protein can quickly cycle between being phosphorylated and O-GlcNAcylated $(10,18)$. An increasing number of kinases have been found to be O-GlcNAcylated, resulting in conformational changes that promote downstream functional effects (11). The important regulatory role of phosphorylation in a subset of proteins associated with transcriptional factor activation, signaling pathways and neurotransmitter synthesis has been identified in OSA-related autonomic abnormalities $(5,8,19)$. It could be suggested that intracellular protein $\mathrm{O}-\mathrm{GlcNAcylation}$ may also play a regulatory role in the OSA-related pathophysiological processes.

Considering the role of O-GlcNAcylation in modulating vascular reactivity and the pathophysiological association between IH and vascular function, the aim of the present study was to investigate whether O-GlcNAcylation exerted regulatory effects on $\mathrm{IH}$ exposure-related vascular dysfunction. In addition, previous evidence has shown that the acute activation of O-GlcNAcylation protects against cellular injury, and that sustained increases in O-GlcNAc proteins may confer deleterious effects on cardiovascular function $(10,12,20)$. Acute IH (AIH), with a hypoxia duration of a few hours, resembles acute I/R injury (1), and the transient elevation of O-GlcNAcylation protects cells against I/R injury (13). On the other hand, chronically-increased levels of O-GlcNAc protein acts as a general mechanism underlying the negative effects of hypertension on vascular function (15). Therefore, the vascular effects of O-GlcNAc modifications on aortic and mesenteric arteries (MAs) subjected to both $\mathrm{AIH}$ and $\mathrm{CIH}$ were investigated. The potential regulatory role of O-GlcNAcylation in p38 MAPK, ERK1/2 and $\mathrm{Ca}^{2+} /$ calmodulin-dependent kinase II (CaMKII) signaling associated with vascular contractile responses during IH exposure were further examined, since these pathways have been found to be targets of O-GlcNAcylation and have been implicated in arterial hypertension-related vascular dysfunction $(5,19,21-28)$. The aim of the present study was to determine whether O-GlcNAcylation contributed to IH-related vascular dysfunction through the modulation of the MAPK and CaMKII signaling pathways. It is hoped that this study could provide novel insights into the molecular mechanisms underlying $\mathrm{CIH}$-associated vascular dysfunction.

\section{Materials and methods}

Animals. All animal experiments were conducted on a total of 90 male Wistar rats provided by the Experimental Animal Center of Tongji Hospital, Huazhong University of Science and Technology (Wuhan, China). Rats were housed with a 12-h light/dark cycle at $22 \pm 1^{\circ} \mathrm{C}$ and $55 \pm 10 \%$ humidity and fed a standard chow diet with ad libitum access to water. All procedures were performed in accordance with the Guide for the Care and Use of Laboratory Animals (29) and approved by the Institutional Animal Care and Use Committee of Huazhong University of Science and Technology. Animal experiments were conducted in the Experimental Animal Center of Tongji Hospital or Tongji Medical College, Huazhong University of Science and Technology.

Reagents and antibodies. Ang II (cat. no. ALX-151-039-M025) and KN-93 (CaMKII inhibitor; cat. no. BML-EI268) were obtained from Enzo Life Sciences, Inc. ST045849 (OGT inhibitor; cat. no. MFCD03308174) was purchased from TimTec, Inc. PD98059 (MEK inhibitor; cat. no. 1213) was from Tocris Bioscience and SB203580 (p38 MAPK inhibitor; cat. no. 13067) from Cayman Chemical Company. Ach (cat. no. A2661), phenylephrine (PE; cat. no. P6126), the NOS blocker N $\omega$-nitro-L-arginine methyl ester (L-NAME; cat. no. N5751), sodium nitroprusside (SNP; cat. no. S0501) and other high-quality reagents were obtained from Sigma-Aldrich (Merck KGaA). PugNAc (OGA inhibitor; cat. no. sc-204415), primary antibodies for O-GlcNAc (mouse monoclonal; cat. no. sc-59623; 1:800), CaMKII (rabbit polyclonal; cat.no. sc-9035; 1:800) and phosphorylated (p)-CaMKII (mouse monoclonal; cat. no. sc-32289; 1:800), as well as secondary anti-mouse (cat. no. sc-358914; 1:4,000) and anti-rabbit IgG antibodies (cat. no. sc-2004; 1:4,000) were purchased from Santa Cruz Biotechnology, Inc. Antibodies against OGT (rabbit polyclonal; cat. no. 11576-2-AP; 1:1,000) and OGA (rabbit polyclonal; cat. no. 14711-1-AP; 1:400) were obtained from ProteinTech Group, Inc. Antibodies against p38 MAPK (rabbit polyclonal; cat. no. 9212; 1:1,000), p-p38 MAPK (rabbit monoclonal; cat. no. 4511; 1:1,000), ERK1/2 (rabbit monoclonal; cat. no. 4695; 1:1,000), p-ERK1/2 (rabbit monoclonal; cat no. 4376; 1:1,000) and GAPDH (rabbit monoclonal; cat. no. 5174; 1:3,000) were purchased from Cell Signaling Technology, Inc. SNP solution was freshly prepared. Stock solutions were prepared either in distilled water (L-NAME, ACh, Ang II and PE) or DMSO (PugNAc, ST045849, SB203580, PD98059 and KN-93), and then frozen in small aliquots. 
AIH exposure. Briefly, a total of 40 12-week-old Wistar rats (weight, 330-370 g) were anesthetized with urethane (1.2 g/kg; i.p.) and euthanized by exsanguination by extracting $\sim 5 \mathrm{ml}$ blood samples with a small needle and syringe following an inferior vena cava puncture. Blood was allowed to flow from the puncture points until the rats lost spontaneous breath and an abdominal aorta pulse (within 3 min). The hearts were harvested and immediately frozen in liquid nitrogen. Thoracic aortas and primary branches of the superior MA (15) were isolated and placed in ice-cold Krebs-Ringer buffer, and connective tissue was removed (5). Next, 4-mm arterial segments were incubated in DMEM (Sigma-Aldrich; Merck KGaA) containing 1\% fetal calf serum (Sigma-Aldrich; Merck KGaA), $120 \mathrm{U} / \mathrm{ml}$ penicillin and $120 \mu \mathrm{g} / \mathrm{ml}$ streptomycin, as previously described (20). To determine the potential regulatory role of O-GlcNAcylation on vascular function, arterial rings were treated with DMSO (vehicle), PugNAc $(100 \mu \mathrm{M})$ or ST045849 $(50 \mu \mathrm{M})$ and exposed to either 3-h normoxic exposure (AON) or 3-h AIH conditions. The incubation time and concentration of ST045849 were determined from pilot studies. The normoxic rings were maintained at atmospheric oxygen concentrations $\left(21 \% \mathrm{O}_{2}, 5 \% \mathrm{CO}_{2}, 37^{\circ} \mathrm{C}\right)$. AIH rings were subjected to 15 alternating cycles of 7 min hypoxia $\left(5 \% \mathrm{O}_{2}, 5 \% \mathrm{CO}_{2}, 37^{\circ} \mathrm{C}\right)$ and 5-min reoxygenation in the chambers attached to a computerized controller (OxyCycler C42; BioSpherix, Ltd.). Thereafter, vessels were either submitted to isometric tension recordings or immediately frozen in liquid nitrogen and stored at $-80^{\circ} \mathrm{C}$ for further protein assays.

CIH procedures. In another set of experiments, a total of 50 8-week-old Wistar rats (weight, 200-250 g) were subjected to either normoxic $\left[21 \% \mathrm{O}_{2}\right.$; control $\left.(\mathrm{CON})\right]$ or $\mathrm{CIH}$ conditions between 8:00 a.m. and 4:00 p.m. for 4 weeks, as previously described (5). During each IH cycle, the oxygen concentration was briefly decreased from 21 to $6-8 \%$ over $40 \mathrm{sec}$ and maintained at $6-8 \%$ for $2 \mathrm{~min}$, which was then increased to $21 \%$ over $20 \mathrm{sec}$ and maintained under normoxia for another 2 min. Normoxia was achieved by compressed air distribution at the same flow rate. After measuring blood pressure, rats were anesthetized with urethane $(1.2 \mathrm{~g} / \mathrm{kg}$, i.p.) and euthanized by exsanguination using inferior vena cava puncture, as described above. Next, rat thoracic aortas and primary MAs were excised, cut into 4-mm segments and subjected to 3-h normoxic incubation with vehicle or antagonists (PugNAc or ST045849).

Vascular reactivity studies. Vascular reactivity was assessed in vessels from the following groups: i) AOD, arterial rings treated with DMSO (vehicle) and 3-h normoxia exposure; ii) AOP, arterial rings treated with PugNAc and 3-h normoxia exposure; iii) AOS, arterial rings treated with ST045849 and 3-h normoxia exposure; iv) AID, arterial rings treated with DMSO and 3-h IH exposure; v) AIP, arterial rings treated with PugNAc and 3-h IH exposure; vi) AIS, arterial rings treated with ST045849 and 3-h IH exposure; vii) COD, arterial rings from control rats treated with DMSO; viii) COP, arterial rings from control rats treated with PugNAc; ix) COS, arterial rings from control rats treated with ST045849; x) CID, arterial rings from $\mathrm{CIH}$ rats treated with DMSO; xi) CIP, arterial rings from $\mathrm{CIH}$ rats treated with PugNAc; and xii) CIS, arterial rings from $\mathrm{CIH}$ rats treated with ST045849. The vascular isometric force was recorded by a $\mathrm{CO}$ pod and PowerLab/4SP data acquisition system (AD Instruments), as previously described $(3-5,30)$. In brief, rings were steadily stretched to optimal passive force (aorta $2 \mathrm{~g}$, MA $1 \mathrm{~g}$ ) established from force-active tension curves to PE $(1 \mu \mathrm{M})$, equilibrated for $45 \mathrm{~min}$ and depolarized with high-K $\mathrm{K}^{+}$Krebs-Ringer buffer (aorta, $120 \mathrm{mM} \mathrm{KCl}$; MA, $80 \mathrm{mM} \mathrm{KCl}$ ). The optimal depolarizing concentration of $\mathrm{KCl}$ was determined using a concentration-response curve (CRC) to $\mathrm{KCl}$ (aorta, 20-140 mM; MA, 20-100 mM) and the contraction generated was regarded as $100 \%$. After a 45 -min washout period, rings were stimulated with PE $(1 \mu \mathrm{M})$ followed by ACh $(10 \mu \mathrm{M})$ to assess the integrity of the endothelium. Next, following a 45-min stabilization period, ACh $(0.001-10 \mu \mathrm{M})$ was added cumulatively to rings with a steady-state maximal contraction induced by PE $(1 \mu \mathrm{M})$. Meanwhile, endothelium-independent relaxation was tested using $\mathrm{SNP}(0.001-3 \mu \mathrm{M})$ in rings pre-incubated with L-NAME $(100 \mu \mathrm{M}, 30 \mathrm{~min})$.

In another subset of segments, CRCs to PE $(0.001-10 \mu \mathrm{M})$ were generated in the presence of DMSO (vehicle) or inhibitors (SB049859, PD98059 or KN-93; $10 \mu \mathrm{M}$ ) added to the Krebs-Ringer buffer for $30 \mathrm{~min}$ before PE stimulation. Each ring was used to generate only one CRC of PE. The endothelium-denuded rings were pretreated with DMSO or the aforementioned inhibitors for $30 \mathrm{~min}$, followed by stimulation with Ang II $(0.1 \mu \mathrm{M})$. Since Ang II might cause tachyphylaxis, a single dose of Ang II was added to each ring (31).

Arterial morphometry and immunohistochemistry (IHC). The tissues were fixed in $4 \%$ formalin for $24 \mathrm{~h}$ at room temperature, processed and embedded in paraffin. Paraffin-embedded sections $(5 \mu \mathrm{m})$ were stained with hematoxylin and eosin (hematoxylin for $\sim 5 \mathrm{~min}$ at room temperature; eosin for $\sim 2$ min at room temperature) and elastin Van Gieson (EVG; $\sim 5 \mathrm{~min}$ at room temperature) to assess arterial wall structure. The vessel luminal diameter (LD), intima-to-media thickness (IMT) and IMT to LD ratio (IMT/LD) were calculated using Image-Pro Plus 7.0 software (IPP; version 7.0; Media Cybernetics, Inc.) (5). EVG stain was used in order to identify elastic fibers by staining them black. The continuity of elastic fibers was observed with a light microscope (magnification, $\mathrm{x} 200$ ). Then, elastic fiber content was determined by calculating the percentage of the positive area to the total cross-sectional vessel wall area using IPP software. An IHC assay was performed using a 3,3'diaminobenzidine (DAB) kit (Agilent Technologies, Inc.), according to the manufacturer's instructions. Briefly, slices were deparaffinized, hydrated, treated with $3 \%$ hydrogen peroxide, blocked in 3\% BSA (Sigma-Aldrich; Merck KGaA) in phosphate-buffered saline ( $\mathrm{pH}$ 7.4) for $30 \mathrm{~min}$ at room temperature, and then incubated with primary antibodies against OGA (1:100) or OGT (1:100) overnight at $4^{\circ} \mathrm{C}$. Following washing, the sections were incubated with HRP-conjugated secondary antibodies (1:200) for $50 \mathrm{~min}$ at room temperature. Slides were washed, developed with DAB, counterstained with hematoxylin for $2 \mathrm{~min}$ at room temperature and mounted with crystal mount. Stained sections were visualized by light microscopy (magnification, x200) and all the images were analyzed using IPP software according to previous study (32). The measurement parameters used to 

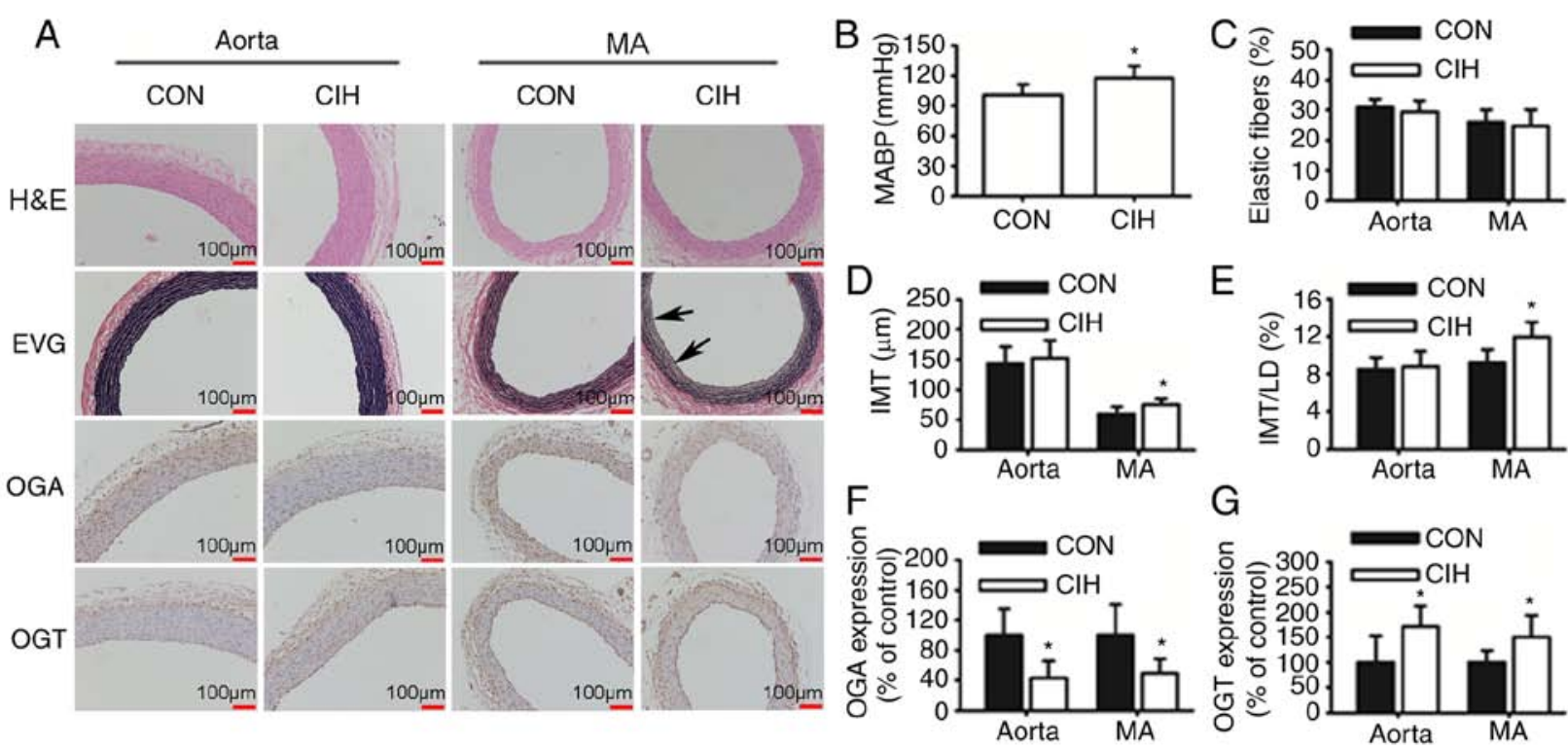

Figure 1. CIH elevates MABP and induces changes in vascular morphology and protein expression. (A) Representative H\&E and EVG staining of vessel walls. Immunohistochemical staining for OGA and OGT. Arrowheads indicate disrupted elastic lamella. (B) MABP was increased by CIH. (C) No differences in the content of elastic fibers were observed. (D and E) Changes in vascular wall thickness. Changes in the protein expression levels of (F) OGA and (G) OGT. Scale bar, $100 \mu \mathrm{m}$. The groups were as follows: i) $\mathrm{CON}$, normoxia $\left(21 \% \mathrm{O}_{2}\right)$; and ii) $\mathrm{CIH}$, intermittent hypoxia cycles $\left(6-8 \% \mathrm{O}_{2}\right.$ for 2 min and $21 \% \mathrm{O}_{2}$ for another $2 \mathrm{~min})$. Data are presented as the mean $\pm \mathrm{SD}(\mathrm{n}=5-20)$. ${ }^{*} \mathrm{P}<0.05$ between groups. $\mathrm{CIH}$, chronic intermittent hypoxia; MABP, mean arterial blood pressure; H\&E, hematoxylin and eosin; EVG, elastin Van Gieson; OGA, O-GlcNAcase; OGT, O-GlcNAc transferase; CON, control; MA, mesenteric artery; LD, luminal diameter; IMT, intima-to-media thickness.

assess the immunostaining quantification were density mean, area sum, and integrated optical density (IOD). The area of interest (AOI) was set for the parameter measurement. The IOD and area sum of AOI was calibrated. Density mean was calculated as the percentage of IOD to area sum and compared between groups.

Western blot analysis. Vascular tissues were homogenized with RIPA buffer containing PMSF ( $1 \mathrm{mM})$, protease inhibitor cocktail and phosphatase inhibitor cocktail. Protein concentration was determined using the Enhanced BCA Protein Assay kit (Beyotime Institute of Biotechnology). Protein extracts $(60 \mu \mathrm{g})$ were separated on $10 \%$ sodium dodecyl sulphate-polyacrylamide gel electrophoresis and transferred onto polyvinylidene fluoride membranes $(0.45 \mu \mathrm{m}$; EMD Millipore) using a Bio-Rad Scientific Instrument (Bio-Rad Laboratories, Inc.). Membranes were blocked with $5 \%$ non-fat milk or 3\% BSA in Tris-buffered saline containing $0.05 \%$ Tween-20 ( $\mathrm{pH} 7.6)$ for $1 \mathrm{~h}$ at room temperature, followed by incubation with primary antibodies specific to O-GlcNAc (1:800), OGT (1:1,000), OGA (1:400), CaMKII (1:800), p-CaMKII (1:800), ERK1/2 (1:1,000), p-ERK1/2 $(1: 1,000)$, p38 MAPK $(1: 1,000)$, p-p38 MAPK $(1: 1,000)$ and GAPDH $(1: 3,000)$ at $4^{\circ} \mathrm{C}$ overnight with gentle shaking. Membranes were incubated with HRP-conjugated secondary antibodies $(1: 4,000)$ for $1 \mathrm{~h}$ at room temperature. The bands were detected using an enhanced chemiluminescence detection kit (Advansta, Inc.). The protein level of GAPDH served as the internal reference. In order to re-use the membrane, stripping buffer (Beyotime Institute of Biotechnology) was used to remove antibodies from the blotted membranes and the membranes were re-probed for other target proteins with similar molecular weight as previously described $(33,34)$. The intensity of bands was semi-quantified using AlphaEaseFC software (version 6.0; ProteinSimple).

Statistical analysis. CRCs to agonists were fitted by non-linear regression using SigmaPlot software (version 11.0; SPSS, Inc.). The maximal responses $\left(\mathrm{E}_{\max }\right)$ and the negative logarithm of the concentration at which agonists produce $50 \%$ of the maximum response were calculated. Statistical analysis was performed using PASW version 18.0 software (SPSS, Inc.). Data are expressed as the mean \pm SD of $\geq 3$ independent repeats. Differences within groups were compared using an unpaired Student's t-test or one-way analysis of variance (ANOVA) followed by LSD post hoc test. $\mathrm{P}<0.05$ was considered to indicate a statistically significant difference.

\section{Results}

CIH causes elevations in mean arterial blood pressure $(M A B P)$ and vascular morphological changes. Rats exposed to $\mathrm{CIH}$ exhibited a higher MABP compared with CON rats (Fig. 1B). The IMT and IMT/LD of MAs were greater in the $\mathrm{CIH}$ group compared with the $\mathrm{CON}$ group. No differences in the IMT and IMT/LD of aortas was observed between the CON and CIH groups (Fig. 1D and E). The elastic lamella of MAs was disrupted by CIH exposure (Fig. 1A). No significant differences in elastic fiber content were observed between the two groups (Fig. 1C). CIH treatment increased the expression of OGT protein but decreased that of OGA in both aortic and mesenteric tissue (Fig. 1F and G).

Protein O-GlcNAc levels influence endothelium-dependent vasodilation during IH exposure. The endothelium-dependent vasodilatory responses of both aortas (Fig. 2A and B) and 

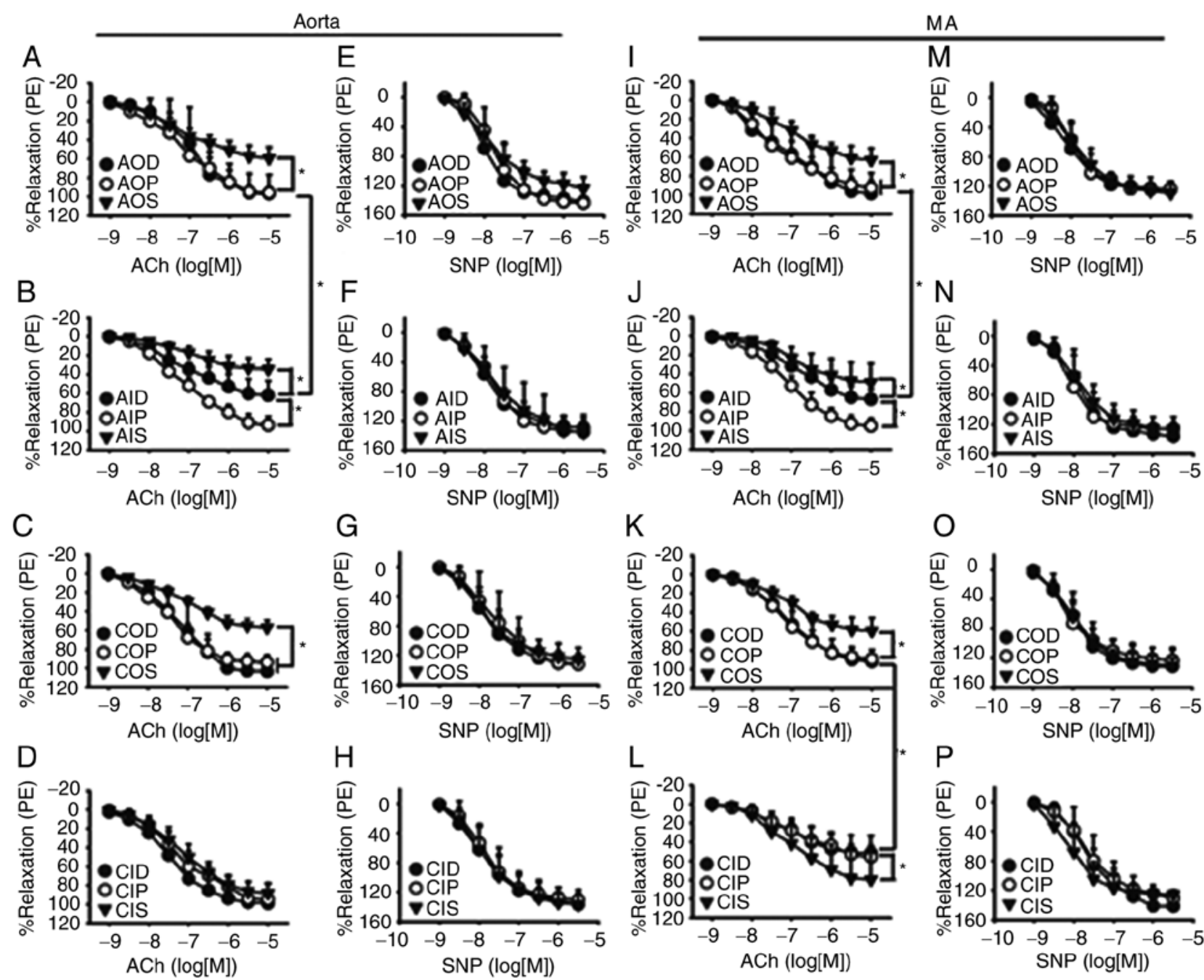

Figure 2. Vasodilatory responses to ACh and SNP of aortas and MAs. O-GlcNAc levels influenced ACh-induced relaxant responses during IH exposure in the (A-D) aorta and (I-L) MA. The vasodilatory responses of both (A and B) aortas and (I and J) MAs to ACh were significantly inhibited by AIH exposure, which was prevented by PugNAc. CIH impaired ACh-induced vasodilation of mesenteric rings, which was partly improved by (K and L) ST045849 but did not have a noticeable effect on the (C and D) aortas. SNP-induced relaxation was similar among all groups in the (E-H) aorta and (M-P) MA. Neither AIH nor $\mathrm{CIH}$ treatment altered SNP-induced relaxation of $(\mathrm{F}$ and $\mathrm{H})$ aortas and (N and P) MAs. The groups were as follows: i) AOD, arterial rings treated with DMSO (vehicle) and 3-h normoxia exposure; ii) AOP, arterial rings treated with PugNAc and 3-h normoxia exposure; iii) AOS, arterial rings treated with ST045849 and 3-h normoxia exposure; iv) AID, arterial rings treated with DMSO and 3-h IH exposure; v) AIP, arterial rings treated with PugNAc and 3-h IH exposure; vi) AIS, arterial rings treated with ST045849 and 3-h IH exposure; vii) COD, arterial rings from control rats treated with DMSO; viii) COP, arterial rings from control rats treated with PugNAc; ix) COS, arterial rings from control rats treated with ST045849; x) CID, arterial rings from CIH rats treated with DMSO; xi) CIP, arterial rings from CIH rats treated with PugNAc; and xii) CIS, arterial rings from CIH rats treated with ST045849. Data are presented as the mean $\pm \mathrm{SD}(\mathrm{n}=5)$. " $\mathrm{P}<0.05$. ACh, acetylcholine; SNP, sodium nitroprusside; O-GlcNAc, O-linked- $\beta$-N-acetylglucosamine; IH, intermittent hypoxia; CIH, chronic intermittent hypoxia; PE, phenylephrine; MA, mesenteric artery.

MAs (Fig. 2I and J) to ACh were significantly inhibited by AIH exposure, which could be prevented by OGA inhibition with PugNAc. CIH impaired the ACh-induced vasodilation of mesenteric rings, which could be partly improved by OGT inhibition with ST045849 (Fig. 2K and L), but did not have a noticeable effect on the aortas (Fig. 2C and D). ST045849 was found to attenuate the $\mathrm{ACh}$-induced relaxation in aortas and MAs that had undergone either normoxia or AIH treatments, except those from the CIH group. SNP-induced endothelium-independent relaxation of aortas (Fig. 2E-H) and MAs (Fig. 2M-P) was similar among all groups .

O-GlcNAcylation modulates contractile responses to Ang II during IH exposure. Neither AIH nor CIH treatment altered vascular reactivity to PE in aortas and MAs. Incubation with
PugNAc or ST045849 did not affect the PE-induced contraction of arteries from any of the studied groups. Of note, SB203580 and PD98059 blocked contractile responses to PE in PugNAc-incubated arteries from the AON, AIH and CON groups. No inhibitory effects of KN-93 on vascular reactivity to PE were observed (Fig. 3).

AIH treatment did not alter contractile responses to Ang II in aortas and MAs, whereas CIH arteries displayed a significantly increased contraction in response to Ang II. The magnitude of Ang II-induced vasoconstriction was not affected by PugNAc intervention. In $\mathrm{CIH}$ arteries that had received vehicle or PugNAc treatment, Ang II-induced contraction was markedly blocked by SB203580 and PD98059, but not by KN-93. A smaller decrease was observed following treatment with PugNAc. Of note, Ang II-induced contraction of 

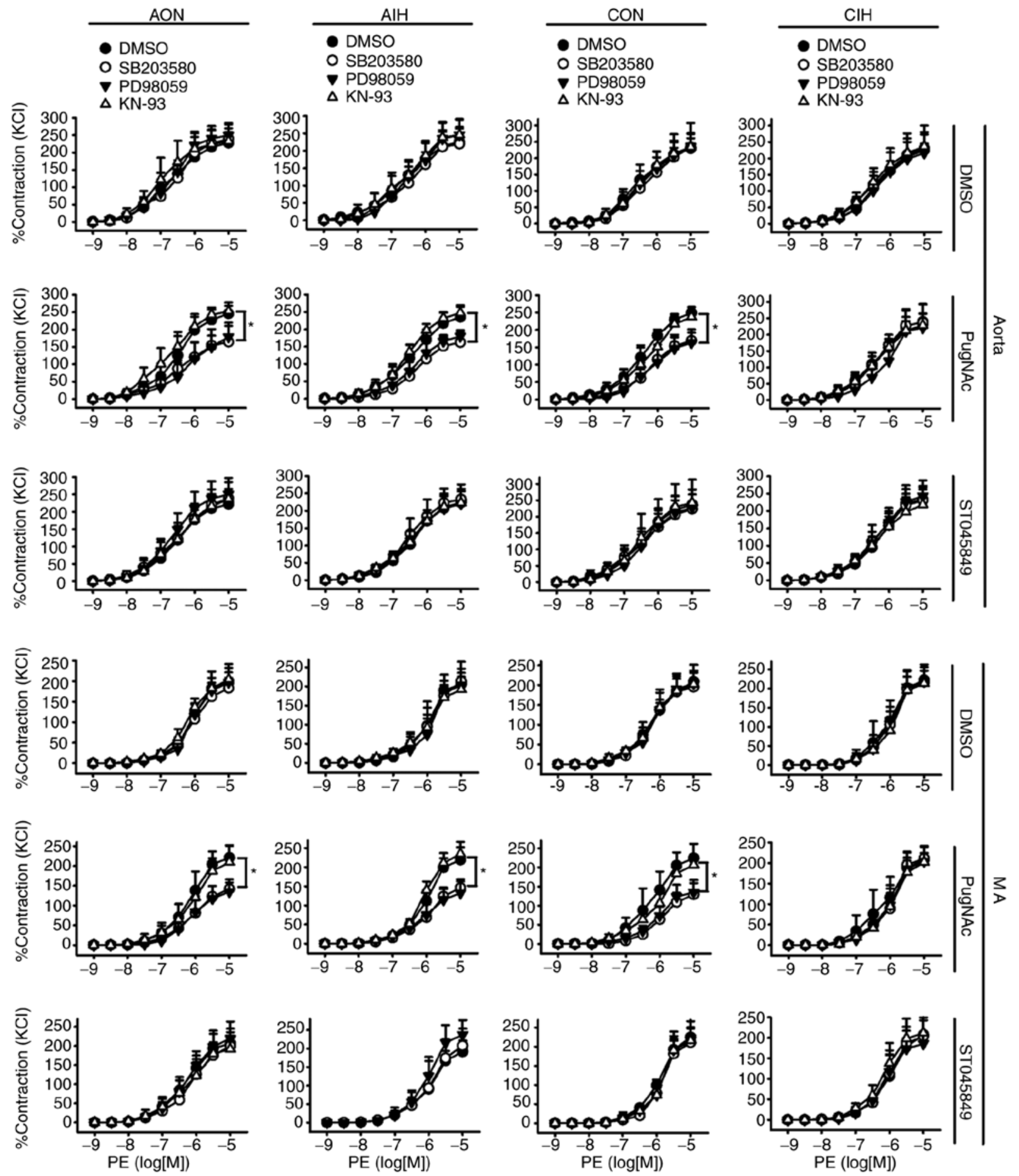

Figure 3. Effects of SB203580, PD98059 and KN-93 on contractile responses to PE in aortas and MAs treated with DMSO, PugNAc or ST045849. Vessels were treated under $\mathrm{AON}$ and $\mathrm{AIH}$ conditions or isolated from $\mathrm{CON}$ and $\mathrm{CIH}$ group rats. The groups were as follows: i) AON, 3-h normoxia treatment; ii) AIH, 3-h intermittent hypxia treatment; iii) CON, normoxic $\left(21 \% \mathrm{O}_{2}\right)$ condition; and iv) $\mathrm{CIH}$, intermittent hypoxia cycles $\left(6-8 \% \mathrm{O}_{2}\right.$ for $2 \mathrm{~min}$ and $21 \% \mathrm{O}_{2}$ for another $2 \mathrm{~min})$. Data are presented as the mean $\pm \mathrm{SD}(\mathrm{n}=5)$. ${ }^{*} \mathrm{P}<0.05$. PE, phenylephrine; AIH, acute intermittent hypoxic; CON, control; $\mathrm{CIH}$, chronic intermittent hypoxia; MA, mesenteric artery.

mesenteric rings from all the non-ST045849-treated groups was inhibited by SB203580 and PD98059, but not by KN-93, with higher inhibitory effects observed in CIH arteries. ST045849 markedly suppressed the contractile responses to Ang II in arteries from all groups, with greater decreases observed in the CIH rings. The inhibitory effects of SB203580 and PD98059 on vascular contraction to Ang II were abolished by ST045849 (Fig. 4).
$O$-GlcNAcylation interferes with the phosphorylation of p38 MAPK, ERK1/2 and CaMKIII. CIH significantly increased the expression of O-GlcNAc and OGT proteins, but reduced the expression of OGA in both aortic and mesenteric tissues (Figs. 1F and G, 5, 6B, S1 and S2B). No statistically significant differences in protein O-GlcNAc levels of cultured aortic rings exposed to $\mathrm{AIH}$ were observed (Fig. 6A and S2A). CIH significantly increased the phosphorylation of p38 MAPK 

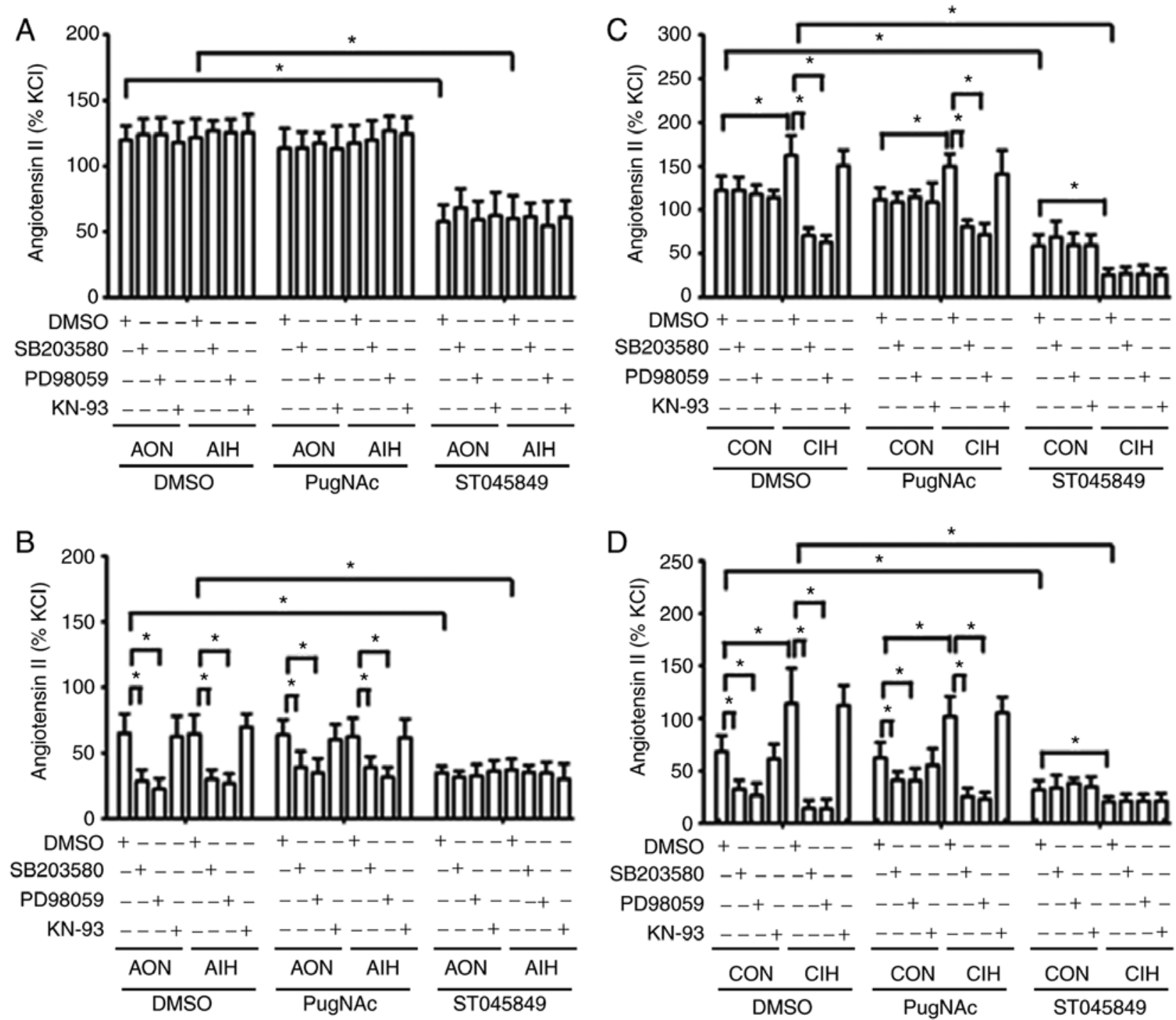

Figure 4. Effects of SB203580, PD98059 and KN-93 on contractile responses to Ang II. Contractile responses to Ang II during AON and AIH exposure in (A) aortic rings and (B) MAs treated with DMSO, PugNAc or ST045849. Ang II-induced contraction of vessels from CON and CIH rats in (C) aortic rings and (D) MAs treated with DMSO, PugNAc or ST045849. The groups were as follows: i) AON, 3-h normoxia treatment; ii) AIH, 3-h intermittent hypxia treatment; iii) $\mathrm{CON}$, normoxic $\left(21 \% \mathrm{O}_{2}\right)$ condition; and iv) $\mathrm{CIH}$, intermittent hypoxia cycles $\left(6-8 \% \mathrm{O}_{2}\right.$ for 2 min and $21 \% \mathrm{O}_{2}$ for another 2 min). Data are presented as the mean $\pm \mathrm{SD}(\mathrm{n}=5) .{ }^{*} \mathrm{P}<0.05$. Ang II, angiotensin II; AIH, acute intermittent hypoxia; CON, control; CIH, chronic intermittent hypoxia; MA, mesenteric artery.

and ERK1/2 but did not exert significant effects on CaMKII phosphorylation levels (Figs. 5, 6B, S1 and S2B). Incubation with PugNAc significantly increased vascular global O-GlcNAcylation, which was accompanied by an increase in the phosphorylation of p38 MAPK and ERK1/2, as well as a slight non-significant elevation in p-CaMKII levels in all groups. On the other hand, ST045849 treatment markedly downregulated O-GlcNAcylation levels, which resulted in a decrease in p38 MAPK and ERK1/2 phosphorylation and no changes in CaMKII phosphorylation (Figs. 6 and S2). No differences in the total expression of p38 MAPK, ERK1/2 and CaMKII were observed among the groups (Figs. 5, 6, S1 and S2).

\section{Discussion}

The overall objective of the present study was to identify the regulatory role of $\mathrm{O}-\mathrm{GlcNAcylation}$ in modulating signaling pathways involved in vascular function during IH exposure. Herein, it was mainly found that protein O-GlcNAc and OGT levels, and the phosphorylation of p38 MAPK and ERK1/2, were increased in aortas and MAs from rats with $\mathrm{CIH}$-induced hypertension. In addition, the OGA levels were decreased, but no changes were observed in p-CaMKII levels. IH exposure could impair endothelial function and vasoconstrictor reactivity. The modulation of global O-GlcNAcylation by pharmacological approaches could interfere with the endothelium-dependent relaxation and vascular contractility, at least partly through MAPK-mediated, but not CaMKII-mediated, signaling pathways.

To the best of our knowledge, the present study was the first to reveal that $\mathrm{CIH}$ exposure significantly increased the content of O-GlcNAc proteins in the vasculature, which was accompanied by upregulated protein levels of OGT and reduced levels of OGA. In addition, elevations in the protein expression levels of O-GlcNAc by $\mathrm{CIH}$ coincided 

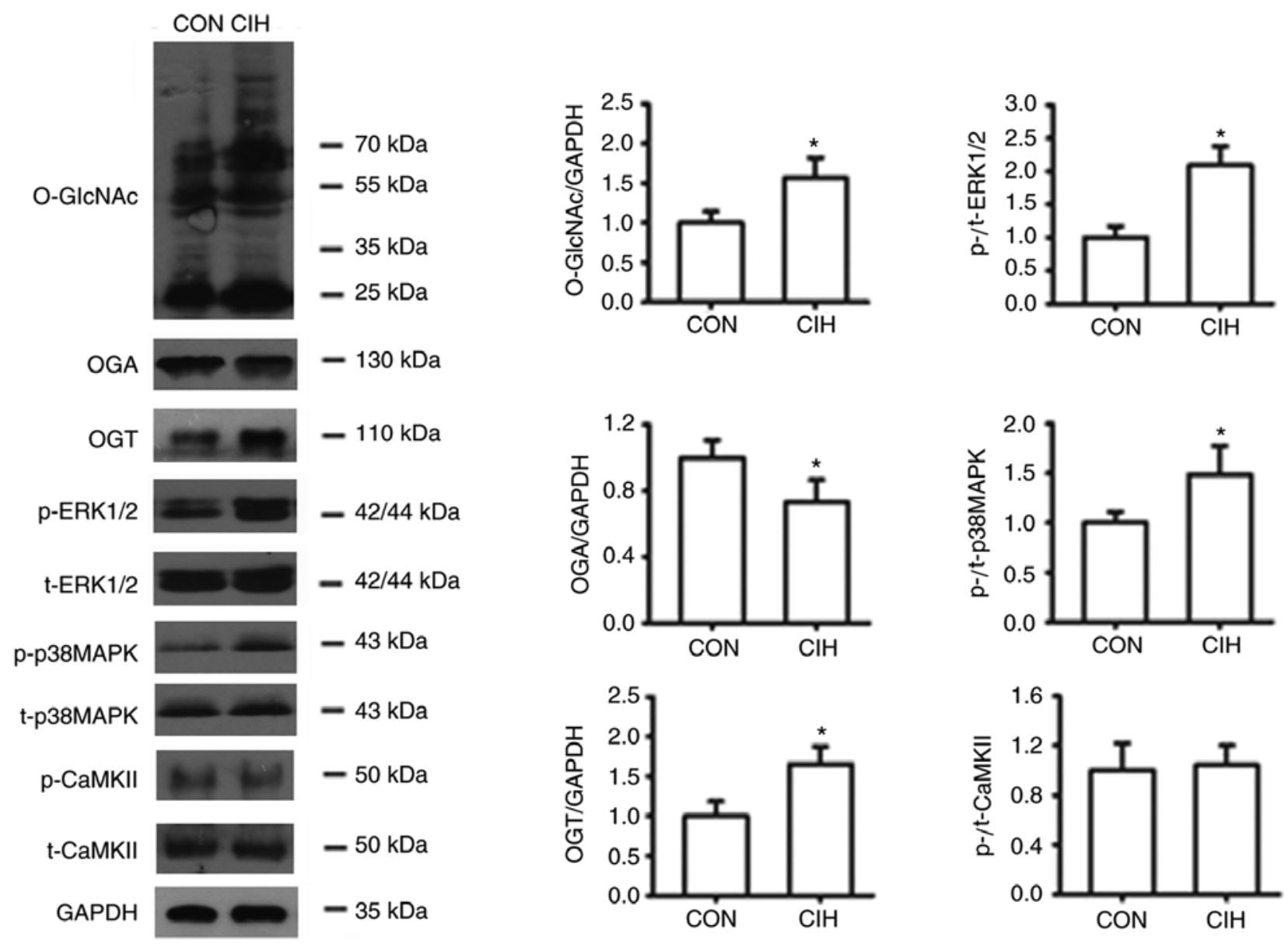

Figure 5. CIH enhances O-GlcNAc levels, and the expression levels of OGT, p-p38 MAPK and p-ERK1/2 levels, but decreases OGA expression in mesenteric arteries. The groups were as follows: i) $\mathrm{CON}$, normoxic $\left(21 \% \mathrm{O}_{2}\right)$ condition; and ii) $\mathrm{CIH}$, intermittent hypoxia cycles $\left(6-8 \% \mathrm{O}_{2}\right.$ for 2 min and $21 \% \mathrm{O}_{2}$ for 2 min). Data are presented as the mean $\pm \mathrm{SD}(\mathrm{n}=3-5)$. $\mathrm{P}<0.05$ vs. CON group. $\mathrm{CIH}$, chronic intermittent hypoxia; O-GlcNAc, O-linked- $\beta-\mathrm{N}$-acetylglucosamine; OGT,

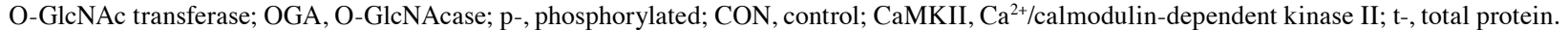

with a decreased endothelium-dependent relaxation in MAs and increased responsiveness to Ang II in aortas and MAs. Similarly, Lima et al (15) reported that O-GlcNAcylation was increased in DOCA-salt hypertensive rats and proposed that sustained increases in O-GlcNAcylation comprise a fundamental mechanism underlying the negative effects of hypertension on vascular function, thereby contributing to hypertension-related vascular dysfunction. In addition, it was discovered that OGA inhibition with the specific inhibitor PugNAc enhanced global O-GlcNAc levels in cultured aortas from all experimental groups, whereas the blockade of OGT with ST045849 had the opposite effects. In addition, PugNAc treatment could protect endothelial cells from AIH-related relaxation injury, but the acute reduction in O-GlcNAcylation by ST045849 tended to restore the relaxant response to ACh in CIH MAs and caused a greater reduction in Ang II-induced contractile responses in $\mathrm{CIH}$ vessels. Collectively, these findings indicated that $\mathrm{IH}$-related vascular dysfunction was, to a certain extent, regulated by O-GlcNAcylation modifications.

In the present study, it was found that $\mathrm{CIH}$ markedly attenuated ACh-induced vasodilation, which was accompanied by increases in the wall thickness of MAs, but not in aortas, which might indicate that the endothelial cells of resistance arteries in the animal models were more susceptible to $\mathrm{CIH}$ insults. $\mathrm{CIH}$-induced endothelial dysfunction most likely results from the decreased generation and bioavailability of NO caused by the unbalanced endothelial expression of eNOS and arginase-1, oxidative stress injury, inflammatory injury, endothelial cell apoptosis and impaired repair process $(3,4,30,35)$. Consistent with these findings, our previous studies revealed that $\mathrm{IH}$ exposure could induce endothelial cell apoptosis due to a disturbance in the $\mathrm{Ca}^{2+}$ homeostasis and endoplasmic reticulum function through the misregulation of the phospholipase $\mathrm{C}$ pathway $(36,37)$. It was also demonstrated in the present study that AIH could impair ACh-induced relaxant responses in both aortic and mesenteric rings, which was prevented by PugNAc incubation. The differences in the functional effects on the endothelium between $\mathrm{AIH}$ and $\mathrm{CIH}$ could be attributed to the relatively weaker anti-oxidant defense and anti-inflammatory capacity of AIH vessels cultured in vitro. In addition, previous data have revealed that acute rises in protein O-GlcNAc levels could improve cellular tolerance to stresses, such as I/R injury, inflammation and oxidative stress $(10,12,20)$. Similarly, as shown in the present study, the increase in protein O-GlcNAc levels could protect human corneal endothelial cells from oxidative stress by attenuating the loss of mitochondrial membrane potential, reducing mitochondrial $\mathrm{Ca}^{2+}$ overload and intracellular reactive oxygen species formation through the Akt signaling pathway (38). Based on these findings, it was hypothesized that the protective effect of PugNAc 
A
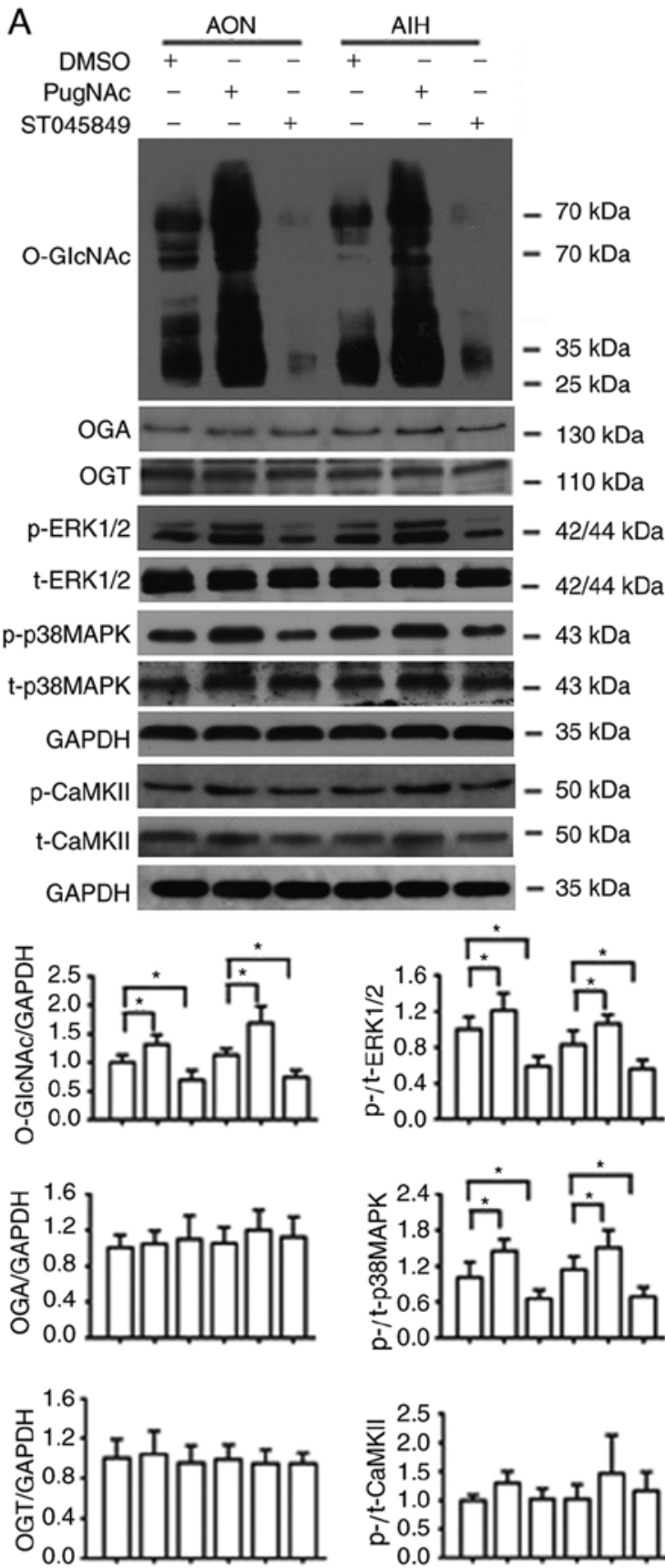

$\mathrm{DMSO}+--+--$

PugNAC -+--+-

ST045849 $\frac{--+}{\mathrm{CON}} \frac{--+}{\mathrm{ClH}}$
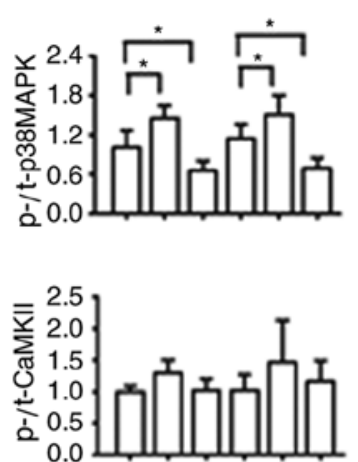

$\mathrm{DMSO}+--+-$

PugNAc -+--+

ST045849
B

\begin{tabular}{|c|c|c|c|c|c|}
\hline \multirow[b]{2}{*}{ DMSO } & \multicolumn{3}{|c|}{$\mathrm{CON}$} & \multicolumn{2}{|c|}{$\mathrm{ClH}$} \\
\hline & + & - & - & + & - \\
\hline PugNAc & - & + & - & - & + \\
\hline ST045849 & - & - & + & - & - \\
\hline
\end{tabular}

O-GIcNAC

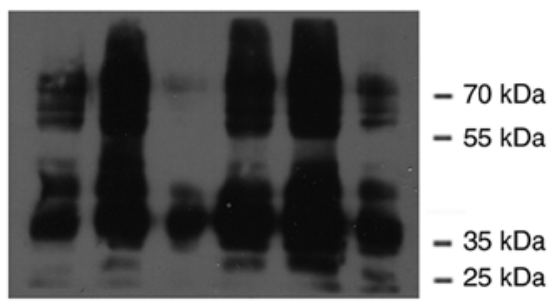

OGA

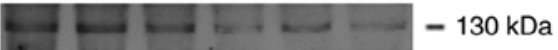

OGT

$-\cdots-$

$-110 \mathrm{kDa}$

p-ERK $1 / 2$

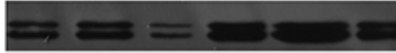

$-42 / 44 \mathrm{kDa}$

t-ERK $1 / 2$

p-CaMKII

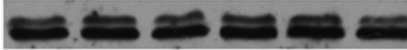

$-42 / 44 \mathrm{kDa}$

t-CaMKII

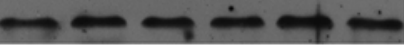

- $50 \mathrm{kDa}$

GAPDH

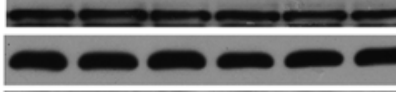

$-50 \mathrm{kDa}$

p-p38MAPK

GAPDH

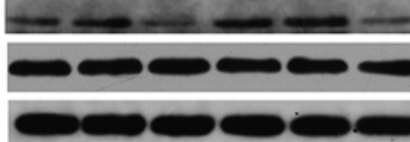

$-35 \mathrm{kDa}$

$-43 \mathrm{kDa}$

$-43 \mathrm{kDa}$
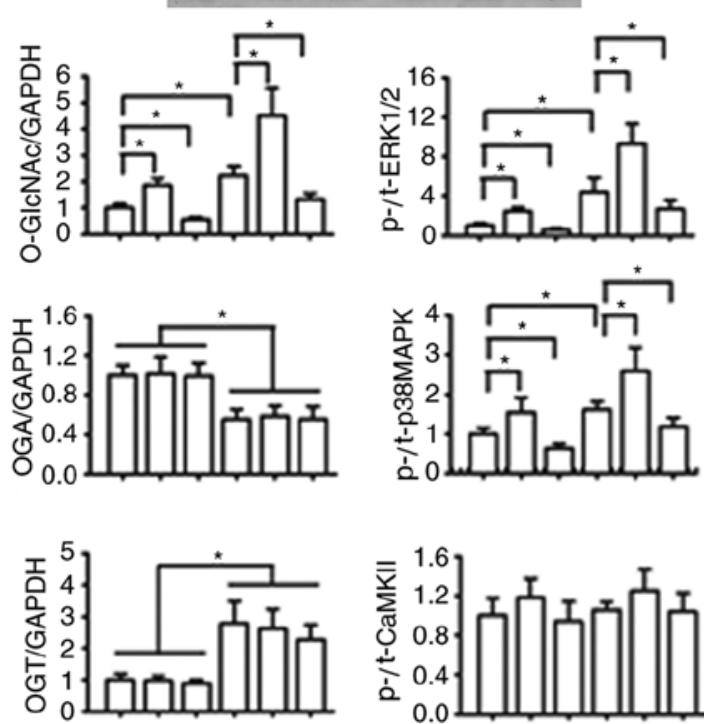

$\mathrm{DMSO}+--+-$

PugNAc - + - - + -

ST045849 - $++\ldots$

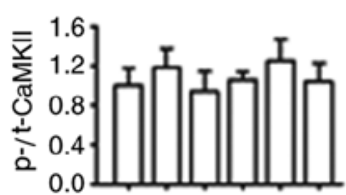

$\mathrm{DMSO}+--+-$

PugNAC -+--+-

ST045849 - $-+-\ldots+$

Figure 6. Protein O-GlcNAc levels interfere with the phosphorylation of p38 MAPK, ERK1/2 and CaMKII. (A) Protein levels of O-GlcNAc, OGA, OGT, p-p38 MAPK, p-ERK1/2 and p-CaMKII in cultured aortas that received either AON or AIH treatment in the presence of DMSO, PugNAc or ST045849. (B) Protein levels of O-GlcNAc, OGA, OGT, p-p38 MAPK, p-ERK1/2 and p-CaMKII in cultured aortas from the CON or CIH rats in the presence of DMSO, PugNAc or ST045849. Experimental groups were as follows: i) AON, 3-h normoxia treatment; ii) AIH, 3-h intermittent hypxia treatment; iii) CON, normoxic $\left(21 \% \mathrm{O}_{2}\right)$ condition; and iv) $\mathrm{CIH}$, intermittent hypoxia cycles $\left(6-8 \% \mathrm{O}_{2}\right.$ for 2 min and $21 \% \mathrm{O}_{2}$ for 2 min). Data are presented as the mean $\pm \mathrm{SD}$ ( $\left.\mathrm{n}=3\right)$. $\mathrm{P}<0.05$. O-GlcNAc, O-linked- $\beta$-N-acetylglucosamine; CaMKII, $\mathrm{Ca}^{2+} /$ calmodulin-dependent kinase II; OGA, O-GlcNAcase; OGT, O-GlcNAc transferase; AIH, acute intermittent hypoxia; CON, control; $\mathrm{CIH}$, chronic intermittent hypoxia; p-, phosphorylated; t-, total protein.

on the endothelium against AIH injury might be associated with its ability to increase O-GlcNAcylated proteins. Of note, reducing global O-GlcNAcylation markedly weakened ACh-induced relaxant responses in vessels under conditions of normoxia and AIH, but tended to improve the relaxation of MAs from $\mathrm{CIH}$ rats, in which vascular O-GlcNAc proteins were chronically increased. Likewise, it has also been revealed that chronically increased O-GlcNAc modification could impair endothelial function by inhibiting eNOS activity and the subsequent release of NO, due to the impaired activation of the PI3K/Akt pathway (25). This, in turn, has been shown to lead to endothelial dysfunction $(10,15,25)$. Therefore, these 
findings further supported the notion that O-GlcNAc formation exerts both protective and adverse effects on vascular function, depending on whether it occurs under acute or chronic stress conditions, respectively, which is most likely through the modulation of different downstream signaling pathways $(13,20)$. Possible regulatory mechanisms involved in the regulation of vascular effects of O-GlcNAcylation during IH exposure require further study.

Regarding the functional consequences of IH on vasoconstriction and signaling mechanisms, it was discovered that $\mathrm{CIH}$ exposure elevated contractile responses to Ang II in both aortas and MAs, but without affecting PE-induced contraction. Similarly, impaired vascular reactivity to different constrictor stimuli, such as Ang II and ET-1, has been reported in the aorta, as well as small mesenteric, cerebral and pulmonary arteries of $\mathrm{CIH}$ animal models $(3,5,7,9,39)$. In individuals with OSA, arterial stiffness, as evaluated by the vasoconstrictor effects of intravenous Ang II challenge, was increased, which could be corrected by continuous positive airway pressure treatment (6). It was also found that the phosphorylation of p38 MAPK and ERK1/2 was increased by CIH, and the blockade of these kinases reduced Ang II-mediated contractions in both $\mathrm{CIH}$ aortic and mesenteric rings. This could be evidenced by previous studies suggesting that MAPKs act as important regulators of vascular reactivity in animal models of hypertension. The activation of ERK $1 / 2$ contributes to elevations in blood pressure, which are most likely associated with the ability of ERK1/2 to modulate vascular oxidative stress, and regulate $\mathrm{Ca}^{2+}$ sensitivity and the downstream contractile protein $(5,23,40,41)$. Furthermore, MAPK inhibition has been reported to block Ang II-induced contraction in MAs from non-ST045849-treated groups $(42,43)$, with higher inhibitory effects observed in $\mathrm{CIH}$ vessels; this might indicate a greater contribution of MAPKs to Ang II-mediated vasoconstriction in small resistance arteries from $\mathrm{CIH}$ rats. These findings supported the present study hypothesis that MAPK pathway activation contributed to $\mathrm{CIH}$-related vascular dysfunction. However, despite evidence that IH could affect CaMKII activity $(44,45)$ and a similar finding showing an increased phosphorylated modification of CaMKII by increased O-GlcNAc levels $(11,22)$, CaMKII blockade did not alter vascular contractile responses to PE or Ang II in any of the groups studied, indicating the lack of CaMKII involvement in the regulation of IH-related vasoconstriction.

A number of kinases or phosphatases can be modified by O-GlcNAcylation, including the CaMK, CK1, tyrosine kinases (TK) and Sterile (STE) kinase families (11). It was further observed in the present study that p38 MAPK and ERK1/2 were phosphorylated in response to enhanced O-GlcNAc levels, whereas reducing O-GlcNAcylation tended to decrease the phosphorylation of these kinases, which was consistent with earlier research showing that O-GlcNAc levels could affect MAPK phosphorylation dynamics. As previously reported, increases in the phosphorylation level of ERK1/2 and p38 MAPK are positively correlated with the increase in O-GlcNAcylation $(26,27)$. Of note, a positive correlation has also been found between activated MAPK/ERK signaling and hyper-O-GlcNAcylation in various cancer types (28). Unlike changes in phosphorylated MAPKs induced by O-GlyNAcylation alterations, the phosphorylation of CaMKII was only slightly enhanced by PugNAc, but this result was not significant, and was not affected by ST045849 in the present study. These findings were consistent with the results of vascular contractile responses. The reason for these findings may have been the differences between the target protein substrates of OGA and those of OGT $(10,11,18)$.

However, no studies have directly addressed how O-GlcNAcylation affects contractile responses in IH-treated vessels. In the current study, it was discovered that Ang II-induced contractions of CIH arteries were attenuated by SB203580 and PD98059, with a lower magnitude of reduction by these inhibitors in vessels treated with PugNAc, possibly due to the relatively higher activity of the kinases following PugNAc incubation. Conversely, ST045849 abolished the inhibitory effects of SB203580 and PD98059 on vasoconstriction to Ang II, suggesting the involvement of other signaling proteins in the regulation of vasoconstrictor sensitivity (46). Based on these observations, it could be concluded that alterations in global O-GlcNAc trigger functional changes in the vasculature during $\mathrm{IH}$ exposure, at least partly by modulating MAPK-mediated signaling pathways. Of note, although PugNAc did not alter PE-induced contraction, MAPK blockade attenuated the contractile response, suggesting the contribution of MAPKs to PE-stimulated vasoconstriction in PugNAc-treated arteries. The alterations in OGA activity may interfere with the contractile apparatus required for PE-induced contraction via MAPK signaling, since MAPK blockade had no effects on vasoconstrictor sensitivity to $\mathrm{PE}$ in the vessels that had not received PugNAc treatment. This could be proven by preliminary data showing that PugNAc increases contractions to PE through the activation of the Ras homolog family member A (RhoA)/Rho-kinase pathway (47). In addition, an interaction between ERK1/2 and Rho-kinase has been reported, where ERK1/2 activation depends on Rho-kinase signaling (48). However, further research is required to confirm this.

The current understanding of the unique molecular $\mathrm{O}-$ GlcNAcylation signaling in regulating vascular reactivity is incomplete. With respect to arterial hypertension and the associated vascular abnormality, several specific pathways, such as PKC, MAPK, PI3K/Akt and RhoA/Rho-kinase, are targets for O-GlcNAcylation $(16,17,21)$. It has been proposed that O-GlcNAc modification of contractile regulators downstream of those specific cascades, such as MLC kinase, MYPT1 and MLC, can interfere with their phosphorylated activities, thus directly influencing vascular smooth muscle function $(16,17,47,49)$.

The present study was not without its limitations. First, changes in the protein levels of OGA, OGT and the phosphorylation of p38 MAPK, ERK1/2 and CaMKII were not investigated in MAs following stimulation with PugNAc or ST045849, but only in aortic tissues, due to the insufficient amount of protein that could be extracted from the MAs. Secondly, the different regulatory role of O-GlcNAcylation in PE-induced and Ang II-induced vasoconstriction could not be determined, possibly due to the agonist-specific signaling transduction cascades $(7,46)$. Thirdly, given the unavailability of specific antibodies for O-GlcNAcylated proteins, which identify the O-GlcNAc-modified sites, the 
exact O-GlcNAcylation levels of MAPKs and CaMKII were not evaluated. Finally, although the in vitro findings suggested pathophysiological links between increased O-GlcNAcylation and $\mathrm{CIH}$-induced vascular dysfunction, future studies using non-toxic pharmacological agents for in vivo experiments, and even transgenic animal models, are required to clarify the exact mechanisms of the regulation of IH-related vascular abnormalities by O-GlcNAcylation.

In conclusion, the present findings demonstrated that $\mathrm{CIH}$ increased global O-GlcNAc proteins in the vasculature and $\mathrm{O}-\mathrm{GlcNAcylation}$ participated in the regulation of misregulated vascular reactivity during $\mathrm{IH}$ exposure, at least partly through MAPK, but not CaMKII signaling pathways. Acute increases in protein O-GlcNAc levels exerted vaso-protective effects against AIH insults, whereas sustained elevations in O-GlcNAc proteins promoted the detrimental effects of $\mathrm{CIH}$ on the vasculature. The modulation of protein O-GlcNAcylation may serve as a therapeutic target for the treatment of impaired vascular function and the associated cardiovascular disorders in patients with OSA.

\section{Acknowledgements}

The authors would like to thank Professor Qinghua Hu from the Department of Pathophysiology, Huazhong University of Science and Technology (Wuhan, China) for the use of the CO pod and PowerLab data acquisition systems.

\section{Funding}

This study was supported by grants from the National Natural Science Foundation of China (grant nos. 81570080 and 81770088) and the Medical and Health Guidance Project of Xiamen (grant no. 3502Z20199018).

\section{Availability of data and materials}

The datasets used and/or analyzed during the current study are available from the corresponding author on reasonable request.

\section{Authors' contributions}

HL, XG and YD contributed to the research design and drafting of the manuscript. XG, YD, LZ and JS performed the experiments. XG, YD and LZ conducted the analysis and interpretation of the data. HL, XG and LZ confirmed the authenticity of all the raw data. All authors have read and approved the final manuscript.

\section{Ethics approval and consent to participate}

All animal procedures were performed in accordance with the Guiding Principles in the Care and Use of Animals and approved by the Institutional Animal Care and Use Committee of Huazhong University of Science and Technology (Wuhan, China).

\section{Patient consent for publication}

Not applicable.

\section{Competing interests}

The authors declare that they have no competing interests.

\section{References}

1. Foster GE, Poulin MJ and Hanly PJ: Intermittent hypoxia and vascular function: Implications for obstructive sleep apnoea. Exp Physiol 92: 51-65, 2007.

2. Cai AP, Wang L and Zhou YL: Hypertension and obstructive sleep apnea. Hypertens Res 39: 391-395, 2016.

3. Krause BJ, Casanello P, Dias AC, Arias P, Velarde V, Arenas GA, Preite MD and Iturriaga R: Chronic intermittent hypoxia-induced vascular dysfunction in rats is reverted by-acetylcysteine supplementation and arginase inhibition. Front Physiol 9: 901-912, 2018.

4. Zhang YN, Zhang CL, Li HO and Hou JD: Down-regulation of vascular PPAR- $\gamma$ contributes to endothelial dysfunction in high-fat diet-induced obese mice exposed to chronic intermittent hypoxia. Biochem Biophys Res Commun 492: 243-248, 2017.

5. Guo XL, Deng Y, Shang J, Liu K, Xu YJ and Liu HG: ERK signaling mediates enhanced angiotensin II-induced rat aortic constriction following chronic intermittent hypoxia. Chin Med J (Engl) 126: 3251-3258, 2013.

6. Nicholl DDM, Hanly PJ, Zalucky AA, Mann MC, MacRae JM, Poulin MJ, Handley GB, Sola DY and Ahmed SB: CPAP therapy delays cardiovagal reactivation and decreases arterial renin-angiotensin system activity in humans with obstructive sleep apnea. J Clin Sleep Med 14: 1509-1520, 2018.

7. Allahdadi KJ, Walker BR and Kanagy NL: ROK contribution to endothelin-mediated contraction in aorta and mesenteric arteries following intermittent hypoxia/hypercapnia in rats. Am J Physiol Heart Circ Physiol 293: H2911-H2918, 2007.

8. Durgan DJ, Crossland RF, Lloyd EE, Phillips SC and Bryan RM: Increased cerebrovascular sensitivity to endothelin-1 in a rat model of obstructive sleep apnea: A role for endothelin receptor B. J Cereb Blood Flow Metab 35: 402-411, 2015.

9. Snow JB, Norton CE, Sands MA, Weise-Cross L, Yan S, Herbert LM, Sheak JR, Gonzalez Bosc LV, Walker BR, Kanagy NL, et al: Intermittent hypoxia augments pulmonary vasoconstrictor reactivity through $\mathrm{PKC} \beta /$ mitochondrial oxidant signaling. Am J Respir Cell Mol Biol 62: 732-746, 2020.

10. Chatham JC, Zhang JH and Wende AR: Role of O-linked $\mathrm{N}$-acetylglucosamine (O-GlcNAc) protein modification in cellular (patho)physiology. Physiol Rev 101: 427-493, 2021.

11. Schwein PA and Woo CM: The O-GlcNAc modification on kinases. ACS Chem Biol 15: 602-617, 2020.

12. Wright JN, Collins HE, Wende AR and Chatham JC: O-GlcNAcylation and cardiovascular disease. Biochem Soc Trans 45: 545-553, 2017.

13. Jensen RV, Andreadou I, Hausenloy DJ and Bøtker HE: The role of O-GlcNAcylation for protection against ischemia-reperfusion injury. Int J Mol Sci 20: 404-424, 2019.

14. Guo XL, Shang J, Deng Y, Yuan X, Zhu D and Liu HG: Alterations in left ventricular function during intermittent hypoxia: Possible involvement of O-GlcNAc protein and MAPK signaling. Int J Mol Med 36: 150-158, 2015.

15. Lima VV, Giachini FR, Choi H, Carneiro FS, Carneiro ZN, Fortes ZB, Carvalho MH, Webb RC and Tostes RC: Impaired vasodilator activity in deoxycorticosterone acetate-salt hypertension is associated with increased protein O-GlcNAcylation. Hypertension 53: 166-174, 2009.

16. Lima VV, Giachini FR, Hardy DM, Webb RC and Tostes RC: O-GlcNAcylation: A novel pathway contributing to the effects of endothelin in the vasculature. Am J Physiol Regul Integr Comp Physiol 300: R236-R250, 2011.

17. Lima VV, Giachini FR, Carneiro FS, Carvalho MH, Fortes ZB, Webb RC and Tostes RC: O-GlcNAcylation contributes to the vascular effects of ET-1 via activation of the RhoA/Rho-kinase pathway. Cardiovasc Res 89: 614-622, 2011.

18. van der Laarse SAM, Leney AC and Heck AJR: Crosstalk between phosphorylation and O-GlcNAcylation: friend or foe. FEBS J 285: 3152-3167, 2018.

19. Kumar GK and Prabhakar NR: Post-translational modification of proteins during intermittent hypoxia. Respir Physiol Neurobiol 164: 272-276, 2008. 
20. Hilgers RHP, Xing DQ, Gong KZ, Chen YF, Chatham JC and Oparil S: Acute O-GlcNAcylation prevents inflammation-induced vascular dysfunction. Am J Physiol Heart Circ Physiol 303: H513-H522, 2012.

21. Lima VV, Rigsby CS, Hardy DM, Webb RC and Tostes RC: O-GlcNAcylation: A novel post-translational mechanism to alter vascular cellular signaling in health and disease: Focus on hypertension. J Am Soc Hypertens 3: 374-387, 2009.

22. Erickson JR, Pereira L, Wang L, Han G, Ferguson A, Dao K, Copeland RJ, Despa F, Hart GW, Ripplinger CM and Bers DM: Diabetic hyperglycaemia activates CaMKII and arrhythmias by O-linked glycosylation. Nature 502: 372-376, 2013.

23. Giachini FR, Sullivan JC, Lima VV, Carneiro FS, Fortes ZB, Pollock DM, Carvalho MHC, Webb RC and Tostes RC: Extracellular signal-regulated kinase $1 / 2$ activation, via downregulation of mitogen-activated protein kinase phosphatase 1 , mediates sex differences in desoxycorticosterone acetate-salt hypertension vascular reactivity. Hypertension 55: 172-179, 2010.

24. Yousif MHM, Akhtar S, Walther T and Benter IF: Role of $\mathrm{Ca}^{2+} /$ calmodulin-dependent protein kinase II in development of vascular dysfunction in diabetic rats with hypertension. Cell Biochem Funct 26: 256-263, 2008.

25. Federici M, Menghini R, Mauriello A, Hribal ML, Ferrelli F, Lauro D, Sbraccia P, Spagnoli LG, Sesti G and Lauro R: Insulin-dependent activation of endothelial nitric oxide synthase is impaired by $\mathrm{O}$-linked glycosylation modification of signaling proteins in human coronary endothelial cells. Circulation 106 466-472, 2002.

26. Goldberg $\mathrm{H}$, Whiteside $\mathrm{C}$ and Fantus IG: O-linked $\beta$-N-acetylglucosamine supports $\mathrm{p} 38$ MAPK activation by high glucose in glomerular mesangial cells. Am J Physiol Endocrinol Metab 301: E713-E726, 2011.

27. Jiang MZ, Qiu ZY, Zhang S, Fan X, Cai XQ, Xu B, Li XW, Zhou JF, Zhang XY, Chu Y, et al: Elevated O-GlcNAcylation promotes gastric cancer cells proliferation by modulating cell cycle related proteins and ERK 1/2 signaling. Oncotarget 7: 61390-61402, 2016.

28. Zhang XL, Ma LN, Qi JQ, Shan H, Yu WG and Gu YC MAPK/ERK signaling pathway-induced hyper-O-GlcNAcylation enhances cancer malignancy. Mol Cell Biochem 410: 101-110, 2015.

29. Institute for Laboratory Animal and Research: Guide for the Care and Use of Laboratory Animals. 8th edition. National Academies Press, Washington, DC, 2011.

30. Li JR, Zhao YS, Chang Y, Yang SC, Guo YJ and Ji ES: Fasudil improves endothelial dysfunction in rats exposed to chronic intermittent hypoxia through RhoA/ROCK/NFATc3 pathway. PLoS One 13: e0195604, 2018.

31. Ishihata A, Tasaki K and Katano Y: Involvement of p44/42 mitogen-activated protein kinases in regulating angiotensin IIand endothelin-1-induced contraction of rat thoracic aorta. Eur J Pharmacol 445: 247-256, 2002.

32. Wang CJ, Zhou ZG, Holmqvist A, Zhang H, Li Y, Adell G and Sun XF: Survivin expression quantified by Image Pro-Plus compared with visual assessment. Appl Immunohistochem Mol Morphol 17: 530-535, 2009

33. Zhu W, Mao Z, Zhu C, Li M, Cao C, Guan Y, Yuan J, Xie G and Guan X: Adolescent exposure to cocaine increases anxiety-like behavior and induces morphologic and neurochemical changes in the hippocampus of adult rats. Neuroscience 313: 174-183, 2016.

34. Zhang Y, Xu Y, Zhu Q, Zhao F, Luo J, Zhang X and Wang X: Upregulation of dysbindin in temporal lobe epileptic foci of human and experimental animals. Synapse 66: 622-629, 2012.

35. Feng J, Zhang D and Chen BY: Endothelial mechanisms of endothelial dysfunction in patients with obstructive sleep apnea. Sleep Breath 16: 283-294, 2012.
36. Yang YY, Shang J and Liu HG: Role of endoplasmic reticular stress in aortic endothelial apoptosis induced by intermittent/persistent hypoxia. Chin Med J (Engl) 126: 4517-4523, 2013.

37. Ren J, Liu W, Deng Y, Li GC, Pan YY, Xie S, Jin M and Liu HG: Losartan attenuates aortic endothelial apoptosis induced by chronic intermittent hypoxia partly via the phospholipase C pathway. Sleep Breath 21: 679-689, 2017.

38. Yoon CK, Yoon SY, Hwang JS and Shin YJ: O-GlcNAc signaling augmentation protects human corneal endothelial cells from oxidative stress via AKT pathway activation. Curr Eye Res 45: $556-562,2020$

39. Friedman JK, Nitta CH, Henderson KM, Codianni SJ, Sanchez L, Ramiro-Diaz JM, Howard TA, Giermakowska W, Kanagy NL and Gonzalez Bosc LV: Intermittent hypoxia-induced increases in reactive oxygen species activate NFATc3 increasing endothelin-1 vasoconstrictor reactivity. Vascul Pharmacol 60: 17-24, 2014.

40. Kim B, Kim J, Bae YM, Cho SI, Kwon SC, Jung JY, Park JC and Ahn HY: p38 mitogen-activated protein kinase contributes to the diminished aortic contraction by endothelin-1 in DOCA-salt hypertensive rats. Hypertension 43: 1086-1091, 2004.

41. Ding LL, Chapman A, Boyd R and Wang HD: ERK activation contributes to regulation of spontaneous contractile tone via superoxide anion in isolated rat aorta of angiotensin II-induced hypertension. Am J Physiol Heart Circ Physiol 292: H2997-H3005, 2007.

42. Escano CS, Keever LB, Gutweiler AA and Andresen BT: Angiotensin II activates extracellular signal-regulated kinase independently of receptor tyrosine kinases in renal smooth muscle cells: Implications for blood pressure regulation. J Pharmacol Exp Ther 324: 34-42, 2008.

43. Matrougui K, Eskildsen-Helmond YE, Fiebeler A, Henrion D, Levy BI, Tedgui A and Mulvany MJ: Angiotensin II stimulates extracellular signal-regulated kinase activity in intact pressurized rat mesenteric resistance arteries. Hypertension 36: 617-621, 2000.

44. Zhang K, Ma ZW, Song C, Duan XR, Yang Y and Li GP: Role of ion channels in chronic intermittent hypoxia-induced atrial remodeling in rats. Life Sci 254: 117797, 2020.

45. Yuan GX, Nanduri J, Bhasker CR, Semenza GL and Prabhakar NR: $\mathrm{Ca}^{2+} /$ calmodulin kinase-dependent activation of hypoxia inducible factor 1 transcriptional activity in cells subjected to intermittent hypoxia. J Biol Chem 280: 4321-4328, 2005.

46. Do KH, Kim MS, Kim JH, Rhim BY, Lee WS, Kim CD and Bae SS: Angiotensin II-induced aortic ring constriction is mediated by phosphatidylinositol 3-kinase/L-type calcium channel signaling pathway. Exp Mol Med 41: 569-576, 2009.

47. Lima VV, Lobato NS, Filgueira FP, Webb RC, Tostes RC and Giachini FR: Vascular O-GlcNAcylation augments reactivity to constrictor stimuli by prolonging phosphorylated levels of the myosin light chain. Braz J Med Biol Res 47: 826-833, 2014.

48. Matrougui K, Tankó LB, Loufrani L, Gorny D, Levy BI, Tedgui A and Henrion D: Involvement of Rho-kinase and the actin filament network in angiotensin II-induced contraction and extracellular signal-regulated kinase activity in intact rat mesenteric resistance arteries. Arterioscler Thromb Vasc Biol 21: 1288-1293, 2001

49. Pedowitz NJ, Batt AR, Darabedian N and Pratt MR: MYPT1 O-GlcNAc modification regulates sphingosine-1-phosphate mediated contraction. Nat Chem Biol 17: 169-177, 2021.

This work is licensed under a Creative Commons Attribution-NonCommercial-NoDerivatives 4.0 International (CC BY-NC-ND 4.0) License. 